\title{
MAN WITH BLURRY VISION
}

Brandy Kaneshiro, $M D,{ }^{1}$ and Albert Yeung, $M D^{2}$

\section{Case Presentation}

A 35 year-old Caucasian man with Pre-B cell ALL on day 197 post allogenic stem cell transplant and with a chest tube for pneumothorax post VATS was admitted for pain at his chest tube site. Sixteen days into his admission he developed acute onset of blurry vision. The patient first noticed some difficulty reading a wall clock when he woke up in the morning followed by a subsequent worsening of his visual acuity over the next eight hours. By late afternoon he could only detect light. Review of systems was significant only for an intermittent headache over the preceding week.

The patient had a past medical history of pre-B cell ALL diagnosed one year prior, allogenic stem cell transplant six months ago, graft versus host disease, nutrition via provided by home TPN, Adenovirus infection three months ago status post IVIG and a hospitalization one month ago during which VATS was performed. The pathology from his VATS was consistent with non-specific interstitial pneumonitis. His hospitalization post-VATS was complicated by pneumothorax and required chest tube placement which was being cared for at home with the assistance of a visiting nurse.

On this admission the patient presented to an outside hospital emergency room complaining of pleuritic chest pain and increasing shortness of breath. Early in his admission he was empirically started on amphotericin B for suspected fungal etiology of his pulmonary symptoms. He was also diagnosed with gastrointestinal graft versus host disease (GVHD) for which he was being treated with steroids and immunosuppressive agents.

Medications included cyclosporine, sirolimus, hydrocortisone, Cellcept, Ambisome, Leukine, amikacin, acyclovir, vancomycin, pentamidine, Dilaudid PCA, acyclovir, Marinol, Nexium, TPN for nutrition, sliding scale insulin and Zoloft.

On physical examination the patient's temperature was $98.3^{\circ} \mathrm{F}$, respiratory rate was 16 , blood pressure was $146 / 107 \mathrm{mmHg}$, pulse was 71 and pulse oximetry was $96 \%$ on room air. His head, neck, heart and lung examination were unremarkable. He had a chest tube to water seal. His abdominal exam was significant for an ileostomy draining brown liquid stool. While he was alert and oriented to person, place and time, he was confused and answered questions inappropriately. His speech was normal and he did not have any focal neurologic deficits. His pupils were equal, round and reactive to light, extra ocular movements could not be assessed secondary to his poor visual acuity and there were no signs of increased intracranial pressure by dilated funduscopic exam.

Laboratory studies on the day of presentation revealed a complete blood count within normal limits. Serum electrolytes and creatinine were unremarkable. Cyclosporine and sirolimus levels were within the appropriate range.

\section{Radiographic studies}

Figures 1-5 are our patient's neuroimaging studies performed the day he presented with rapid vision loss. While there is no single pathognomic radiographic finding for PRES, these CT and MRI images demonstrate a series of findings typically suggestive of the syndrome.

The clinical presentation above confirmed by MRI led to an early diagnosis of Posterior Reversible Encephalopathy Syndrome (PRES) in our patient. The most likely culprit was the cyclosporine he had been taking post stem cell transplant. Both his cyclosporine and sirolimus were stopped immediately. Our patient's vision loss persisted four days after the cyclosporine and sirolimus were discontinued. On the fifth day, however, he woke in the morning reporting a dramatic, complete resolution of his visual disturbances.

\section{Discussion}

Posterior Reversible Encephalopathy Syndrome (PRES)

The classic clinical syndrome includes headache, visual changes, confusion, lethargy and seizures. The headache is typically described as constant, bilateral, moderate and dull in quality. Visual changes can progress to complete visual loss or may manifest as visual hallucinations. Fundoscopic exam is usually normal as was seen in our patient. Neurologic exam rarely reveals any focal deficits. Seizures usually present as generalized tonic clonic.

Little can yet be confirmed about the incidence of or risk factors for this syndrome since a significant portion of what is known is based on published case reports. PRES is most commonly seen as a complication of hypertensive encephalopathy, eclampsia and cytotoxic and immunosuppressant medications (most notably cyclosporine, tacrolimus and interferon) but there is a long list of

${ }^{1}$ Thomas Jefferson University Hospital, Philadelphia, PA

${ }^{2}$ Hahnemann University Hospital, Philadelphia, PA 


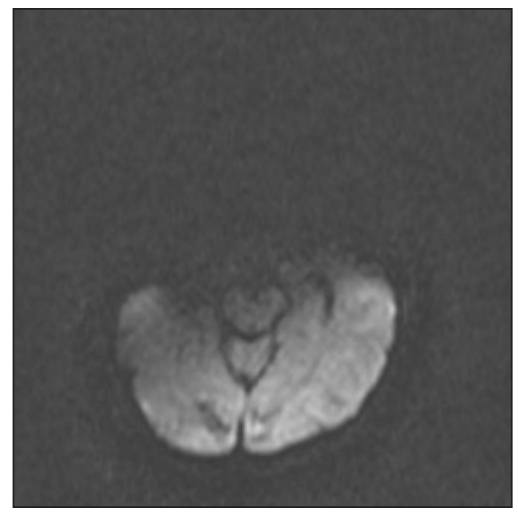

Figure 3. Diffusion weighted image of the occipital lobes is normal. Cytotoxic edema secondary to tissue ischemia is excluded with this sequence.

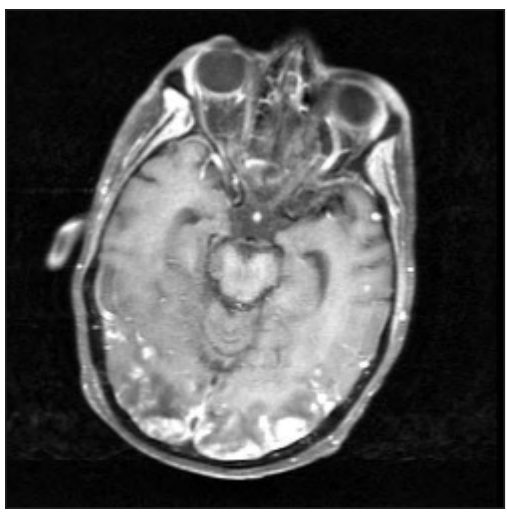

Figure 4. Post-contrast axial T1 weighted image shows patchy enhancement of the occipital lobes, consistent with disruption of the blood brain barrier.

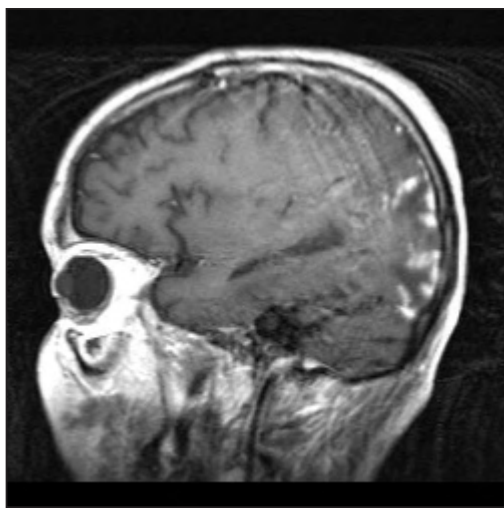

Figure 5. Post-contrast sagittal T1 weighted image demonstrates patchy enhancement of the occipital lobe. documented etiologies. These varying clinical scenarios are most clearly unified by the neuroimaging findings seen on CT scanning and MRI. While cyclosporine is the most common medication associated with PRES, it is unclear what specific trigger initiates the syndrome in any particular patient. PRES can develop within days or many months after exposure and serum levels of cyclosporine also seem to be a poor predictor of risk for the syndrome.

There are multiple hypotheses concerning the pathogenesis of PRES but most of the literature describes the syndrome as the result of impaired cerebral autoregulation and endothelial dysfunction. The most plausible hypothesis is that, as the body's ability to maintain constant cerebral perfusion is impaired or exceeded, increases in systemic blood pressure could lead to extravasation of fluid into the surrounding tissue causing vasogenic edema. Endothelial dysfunction may also contribute since PRES seems to occur with higher frequency in patients with chronic renal failure, vasculitis, sepsis and hemolytic uremic syndrome.

Care must be taken to exclude other equally serious diagnoses such as embolic stroke, venous thrombosis, metabolic encephalopathy, encephalitis, vasculitis or demyelinating disorders. This can best be accomplished with neuroimaging including CT scanning and MRI. Most frequently seen is symmetrical white matter edema in the parieto-occipital regions as noted in Figures 1-5.

Appropriate treatment requires discontinuation of the offending agent or treatment of the underlying disease. Despite optimal care, some patients sustain permanent vision loss or epilepsy secondary to PRES. In the majority of cases, however, clinicians can expect resolution of PRES in days to weeks. Blood pressure should be tightly maintained in the normal range with titratable parenteral agents such as nicardipine or labetalol. Current guidelines for treating malignant hypertension should be incorporated into the management of the patient when applicable. Steroids are used in some settings but are not widely accepted as an appropriate treatment because of the associated risk of worsening hypertension and fluid overload. Neuroimaging will also document resolution of PRES correlating well with the patient's symptoms. The offending agent should never be reintroduced to the patient.

The acute development of seizures, visual disturbances, headache or confusion especially in a patient with hypertension, taking immunosuppressive or cytotoxic agents or with impaired renal function should prompt an urgent CT scan and MRI to evaluate for PRES. This syndrome, while usually reversible in days to weeks following treatment of the underlying process or withdrawal of the culprit medication, has the potential to remain permanent resulting in significant disability if there is a delay in appropriate diagnosis and treatment.

\section{References}

1. Shin K, Choi HJ, Bae YD, et al. Reversible posterior leukoencephalopathy syndrome in systemic lupus erythematosus with thrombocytopenia treated with cyclosporine. J Clin Rheum 2005; 11(3):164-6.

2. Tam CS, Galanos J, Seymour JF, et al. Reversible posterior leukoencephalopathy syndrome complicating cytotoxic chemotherapy for hematologic malignancies. Am J Hemat 2004; 77(1):72-6.

3. Mukherjee P, McKinstry RC. Reversible posterior leukoencephalopathy syndrome: evaluation with diffusion-tensor MR imaging. Radiology 2001; 219(3):756-65.

4. Stott, VL, Hurrell, MA, Anderson, TJ. Reversible posterior leukoencephalopathy syndrome: a misnomer reviewed. Intern Med J 2005; 35:83.

5. Schwartz, RB, Jones KM, Kalinea, P, et al. Hypertensive encephalopathy: findings on CT, MR imaging and SPECT imaging in 14 cases. Am J Roentgenol 1992; 159:379. 